\title{
Guo's index for some classes of matrices
}

\author{
María Robbiano \\ Departamento de Matemáticas, Facultad de Ciencias. Universidad Católica del Norte. \\ Av. Angamos 0610 Antofagasta, Chile.
}

\begin{abstract}
A permutative matrix is a square matrix such that every row is a permutation of the first row. A circulant matrix is a matrix where each row is a cyclic shift of the row above to the right. The Guo's index $\lambda_{0}$ of a realizable list is the minimum spectral radius such that the list (up to the initial spectral radius) together with $\lambda_{0}$ is realizable. The Guo's index of some permutative matrices is obtained. Our results are constructive. Some examples designed using MATLAB are given at the end of the paper.
\end{abstract}

Keywords: Inverse eigenvalue problem; Structured inverse eigenvalue problem; Circulant matrix; Permutative matrix; Guo index 2000 MSC: 15A18, 15A29, 15B99.

\section{Preliminaries}

In this section we present a brief resume. Recall that a square matrix $A=$ $\left(a_{i j}\right)$ is nonnegative $(A \geq 0)$ if and only if $a_{i j} \geq 0 \quad(1 \leq i, j \leq n)$. A list is an $n$-tuple, $\Lambda=\left(\lambda_{1}, \ldots, \lambda_{n}\right)$ of complex numbers and it is realized by a $n$-by $n$ nonnegative matrix $A$ if the set formed its components and the spectrum of $A$ (considering multiplicities) coincide. The NIEP is the problem of determining necessary and sufficient conditions for a list of $n$ complex numbers to be realized by an $n$-by- $n$ nonnegative matrix $A$. If a list $\Lambda$ is realized by a nonnegative matrix $A$, then $\Lambda$ is realizable and the matrix $A$ realizes $\Lambda$ (or, that is a realizing matrix for the list). Some results can be seen in e.g. [10, 11, 12]. A variant of the original problem is the question for which lists of $n$ real numbers can occur as eigenvalues of an $n$-by- $n$ nonnegative matrix

Email address: mrobbiano@ucn.cl (María Robbiano) 
and it is called real nonnegative inverse eigenvalue problem (RNIEP). Some results can be seen in e.g. [8]. The structured NIEP is an analogous problem to NIEP where the realizing matrix must be structured, for instance, the matrix can be symmetric, Toeplitz, Hankel, circulant, normal, permutative, etc., see in [5, 8, 13, 15, 16] and the reference therein. In this paper we deal with structured matrices. In particular, permutative and circulant matrices. Throughout the text, $\sigma(A)$ denotes the set of eigenvalues of a square matrix $A$. As usual, the identity matrix of order $n$ is denoted by $I_{n}$ and if the order of the identity matrix can be easily deduced then it is just denoted by $I$.

Since a nonnegative matrix is real, its characteristic polynomial must have real coefficients and then $\left\{\lambda_{0}, \ldots, \lambda_{n-1}\right\}=\Lambda=\bar{\Lambda}=\left\{\overline{\lambda_{0}}, \ldots, \overline{\lambda_{n-1}}\right\}$, where $\bar{\lambda}$ stands for the complex conjugate of $\lambda \in \mathbb{C}$.

Therefore consider the following definition:

Definition 1. The complex n-tuple $\left(\lambda_{0}, \ldots, \lambda_{n-1}\right)$ is closed under complex conjugation if the condition

$$
\left\{\lambda_{0}, \ldots, \lambda_{n-1}\right\}=\left\{\overline{\lambda_{0}}, \ldots, \overline{\lambda_{n-1}}\right\},
$$

holds.

The Perron-Frobenius theory of nonnegative matrices [2] plays in this problem an important role. The theory provides several important necessary conditions for the NIEP. See below some of these conditions resumed. Here, for $1 \leq k \leq n, \quad s_{k}(\Lambda)=\sum_{i=0}^{n-1} \lambda_{i}^{k}$ is named the $k$-th moment.

Some necessary conditions for the list $\Lambda=\left(\lambda_{0}, \ldots, \lambda_{n-1}\right)$ of complex numbers to be the spectrum of a nonnegative matrix are:

1. The spectral radius, $\max \{|\lambda|: \lambda \in \Lambda\}$, called the Perron eigenvalue, belongs to $\Lambda$.

2. The list $\Lambda$ is closed under complex conjugation.

3. $s_{k}(\Lambda) \geq 0, k \geq 1$.

4. $s_{k}^{m}(\Lambda) \leq n^{m-1} s_{k m}(\Lambda), k \geq 1$.

The last condition was proved by Johnson [7] and independently by Loewy and London [14].

The following fundamental theorem was proven in [6] and in its statement it is introduced formally the notion of Guo's index. 
Theorem 2. [6, Theorem 2.1] Let $\left(\lambda_{1}, \ldots, \lambda_{n-1}\right)$ be a closed under complex conjugation $(n-1)$-tuple then, there exists a real number $\lambda_{0}$ (called Guo 's index) where

$$
\max _{1 \leq j \leq n-1}\left|\lambda_{j}\right| \leq \lambda_{0} .
$$

such that the list $\left(\lambda, \lambda_{1}, \ldots, \lambda_{n-1}\right)$ is realizable by an $n$-by-n nonnegative matrix $A$ if and only if $\lambda \geq \lambda_{0}$. Furthermore, $\lambda_{0} \leq 2 n \max _{1 \leq j \leq n-1}\left|\lambda_{j}\right|$.

We define permutative matrix, below.

Definition 3. [16] A square matrix of order $n$ with $n \geq 2$ is called a permutative matrix or permutative when all its rows (up to the first one) are permutations of precisely its first row.

The spectra of a class of permutative matrices was studied in [15]. In particular, spectral results for matrices partitioned into 2-by-2 symmetric blocks were presented and, using these results sufficient conditions on a given list to be the list of eigenvalues of a nonnegative permutative matrix were obtained and the corresponding permutative matrices were constructed.

This paper is structured in 4 sections. In the second section we obtain necessary and suficient conditions for a list $\Lambda$ to be the spectrum of a class of permutative matrices. In the third section results about circulant and block matrices, given in [1] are revisited. In the fourth section necessary and sufficient conditions for some lists to be realized by a class of structured matrices are exhibited.

\section{Classes of Permutative Matrices}

In this section some auxiliary results from [16] are recalled and some new definitions are introduced. In [16] the following results were proven.

Lemma 4. [16, Lemma 3.1] For $\mathbf{x}=\left(x_{1}, x_{2}, \ldots, x_{n}\right)^{T} \in \mathbb{C}^{n}$, let

$$
X=\left(\begin{array}{ccccccc}
x_{1} & x_{2} & \ldots & x_{i} & \ldots & x_{n-1} & x_{n} \\
x_{2} & x_{1} & \ldots & x_{i} & \ldots & x_{n-1} & x_{n} \\
\vdots & \vdots & \ddots & \vdots & \ddots & \vdots & \vdots \\
x_{i} & x_{2} & \ddots & x_{1} & \ddots & \vdots & \vdots \\
\vdots & \vdots & \vdots & \vdots & \ddots & \vdots & \vdots \\
x_{n-1} & x_{2} & \ldots & \vdots & \vdots & x_{1} & x_{n} \\
x_{n} & x_{2} & \ldots & x_{i} & \ldots & x_{n-1} & x_{1}
\end{array}\right) .
$$


Then, the set of eigenvalues of $X$ is given by

$$
\sigma(X)=\left\{\sum_{i=1}^{n} x_{i}, x_{1}-x_{2}, x_{1}-x_{3}, \ldots, x_{1}-x_{n}\right\} .
$$

Definition 5. The list $\Lambda=\left(\lambda_{1}, \ldots, \lambda_{n}\right)$ is a Suleimanova spectrum if the $\Lambda$ is real list, $\lambda_{1}>0 \geq \lambda_{2} \geq \cdots \geq \lambda_{n}$ and $\sum_{i=1}^{n} \lambda_{i} \geq 0$.

Theorem 6. [16] Let $\Lambda=\left(\lambda_{1}, \ldots, \lambda_{n}\right)$ be a Sulermanova spectrum and consider the $n$-tuple $\mathbf{x}=\left(x_{1}, x_{2}, \ldots, x_{n}\right)$, where

$$
x_{1}=\frac{\lambda_{1}+\cdots+\lambda_{n}}{n} \text { and } x_{i}=x_{1}-\lambda_{i}, 2 \leq i \leq n,
$$

then the matrix in (1) realizes $\Lambda$.

Lemma 7. 16] Let $\mathbf{e}$ and $J$ be the all ones column vector and the all ones square matrix, respectively. Let

$$
M=\left(\begin{array}{cc}
1 & \mathbf{e}^{T} \\
\mathbf{e} & -I_{n-1}
\end{array}\right)
$$

then

$$
M^{-1}=\frac{1}{n}\left(\begin{array}{cc}
1 & \mathbf{e}^{T} \\
\mathbf{e} & J-n I_{n-1}
\end{array}\right)
$$

The following fact is an immediate consequence of Lemmas 4 and 7 .

Remark 8. Let $\Lambda=\left(\lambda_{1}, \ldots, \lambda_{n}\right)$ and consider $\mathbf{x}^{\mathbf{T}}=M^{-1} \Lambda^{T}$ then $\Lambda$ is the list of eigenvalues of the matrix $X$ in (1), where $\mathbf{x}=\left(x_{1}, \ldots x_{n}\right)$.

The following notions will be used in the sequel.

Definition 9. Let $\tau=\left(\tau_{1}, \ldots, \tau_{n}\right)$ be an $n$-tuple whose elements are permutations in the symmetric group $S_{n}$, with $\tau_{1}=i d$. Let $\mathbf{a}=\left(a_{1}, \ldots, a_{n}\right) \in \mathbb{C}^{n}$. Define the row-vector,

$$
\tau_{j}(\mathbf{a})=\left(a_{\tau_{j}(1)}, \ldots, a_{\tau_{j}(n)}\right)
$$

and consider the matrix

$$
\tau(\mathbf{a})=\left(\begin{array}{lllll}
\tau_{1}(\mathbf{a}) & \tau_{2}(\mathbf{a}) & \ldots & \tau_{n-1}(\mathbf{a}) & \tau_{n}(\mathbf{a})
\end{array}\right)^{T} .
$$

A square matrix $A$, is called $\tau$-permutative if $A=\tau(\mathbf{a})$ for some $n$-tuple a. 
Definition 10. [15] If $A$ and $B$ are $\tau$-permutative by a common vector $\tau=\left(\tau_{1}, \ldots, \tau_{n}\right)$ then they are called permutatively equivalent.

We now need to define the following concept.

Definition 11. If $A$ and $B$ are permutatively equivalent matrices then $B$ is A-like permutative or $A$ is $B$-like permutative.

Remark 12. A permutative matrix A defines the class of permutatively equivalent matrices: The class of the A-like permutative matrices. Let $\sigma_{1}$ be an arbitrary Suleimanova spectra, then the corresponding realizing matrix $X_{\sigma_{1}}$ given by Theorem [6 is a $X$-like permutative matrix, where $X$ is as the matrix in (11). Furthermore, by Lemma 4 and Remark 8 it is easy to check that given an arbitrary n-tuple (not necessarily into the NIEP) there exist a solution which is $X$-like permutative, where $X$ is as the matrix in (1). By simplicity, during the paper we use $X$-like permutative matrix to refer a $X$-like permutative matrix, where $X$ is as the matrix in (11).

The following definition concerns to the spectra.

Definition 13. Given the list $\Lambda=\left(\lambda_{0}, \lambda_{1}, \lambda_{2}, \ldots, \lambda_{n-1}\right)$ we say that $\Lambda$ is $X$ like permutative list if there exists a nonnegative $X$-like permutative matrix whose spectrum is $\Lambda$.

By Theorem [6 the Suleumanova lists are $X$-like permutative. Also the list $\Lambda=(24,5,6,7,8)$ is a $X$-like permutative list as the $X$-like permutative matrix whose first row is $(10,2,3,4,5)$ realizes $\Lambda$.

Remark 14. By the equations in Theorem [, a X-like permutative spectrum is always a real spectrum.

The following result is a necessary and sufficient condition for the list $\Lambda=$ $\left(\lambda_{1}, \lambda_{2}, \ldots, \lambda_{n}\right)$ to be a $X$-like permutative list.

Theorem 15. Let $\Lambda=\left(\lambda_{1}, \lambda_{2}, \ldots, \lambda_{n}\right)$, be a real list, where $\lambda_{1}=\max _{2 \leq \ell \leq n}\left|\lambda_{\ell}\right|$, and $\sum_{j=1}^{n} \lambda_{j} \geq 0$. Then the set formed by the components of $\Lambda$ are the eigenvalues of a nonnegative irreducible $X$-like permutaitive matrix if and only if

$$
\lambda_{1}+(n-1) \lambda_{\nu(i)} \geq \sum_{j=2, j \neq i}^{n} \lambda_{\nu(j)}
$$


for all $2 \leq i \leq n$ and for some $\nu \in S_{n-1}$, the symmetric group of the permutations of the set $\{2, \ldots, n\}$. In consequence, the expression

$$
\min _{\nu \in S_{n-1}} \max _{2 \leq i \leq n} \sum_{j=2,}^{n} \lambda_{\nu(j)}-(n-1) \lambda_{\nu(i)}
$$

is a Guo index for X-like permutative lists.

Proof. If there exits a permutation $\nu \in S_{n-1}$ such that the inequality in (4) holds then the vector $\mathbf{x}^{\mathbf{T}}=M^{-1} \Lambda_{\nu}^{\mathbf{T}}$, is nonnegative, where

$$
\boldsymbol{\Lambda}_{\nu}=\left(\lambda_{1}, \lambda_{\nu(2)}, \ldots, \lambda_{\nu(n)}\right)
$$

by the matrix equations in Remark 8 we conclude that a nonnegative $X$ like permutative matrix whose first row is $\mathbf{x}$ has the components of $\Lambda$ as eigenvalues. Reciprocally, if the components of $\Lambda$ are the eigenvalues of a nonnegative $X$-like permutative matrix whose first row is $\mathbf{x}$, by Remark 8 $\mathbf{x}^{T}=M^{-1} \Lambda_{\mu}^{T}$ for some permutation $\mu$ in $S_{n-1}$, then by the non-negativity of $\mathbf{x}$ the inequalities in (4) hold for the permutation $\mu$.

By simplicity, during the paper, a $X$-like permutative matrix whose first row is $\mathbf{x}=\left(x_{1}, \ldots, x_{n}\right)$ is written

$$
\operatorname{per}_{X}\left(x_{1}, \ldots, x_{n}\right) \quad \text { or } \operatorname{per}_{X}(\mathbf{x}) .
$$

For $\tau$-permutative or - -like permutative matrices an analogous property of circulant matrices was proven in [15].

Proposition 16. Let $\left\{A_{i}\right\}_{i=1}^{k}$ be a family of pairwise permutatively equivalent square matrices. Let $\left\{\gamma_{i}\right\}_{i=1}^{k}$ be a set of complex numbers. Consider

$$
A=\sum_{i=1}^{k} \gamma_{i} A_{i}
$$

Then $A$ is a $A_{1}$-like permutative matrix. 


\section{Matrices partitioned into circulant blocks}

The class of circulant matrices and their properties are introduced in [9]. Let $\mathbf{a}=\left(a_{0}, a_{1}, \ldots, a_{m-1}\right)$ be $m$-tuple of complex numbers.

Definition 17. [9] A real circulant matrix is a matrix of the form

$$
\operatorname{circ}(\mathbf{a})=\left(\begin{array}{ccccc}
a_{0} & a_{1} & \ldots & \ldots & a_{m-1} \\
a_{m-1} & a_{0} & a_{1} & \ldots & a_{m-2} \\
a_{m-2} & \ddots & \ddots & \ddots & \vdots \\
\vdots & \ddots & \ddots & a_{0} & a_{1} \\
a_{1} & \ldots & a_{m-2} & a_{m-1} & a_{0}
\end{array}\right)
$$

The next concepts can be seen in [9]. The entries of the unitary discrete Fourier transform (DFT) matrix $F=\left(f_{p q}\right)$ are given by

$$
f_{p q}=\frac{1}{\sqrt{n}} \omega^{p q}, \quad 0 \leq p, q \leq m-1,
$$

where

$$
\omega=\cos \frac{2 \pi}{m}+i \sin \frac{2 \pi}{m} .
$$

The following results characterize the circulant spectra.

Theorem 18. [9] Let $\mathbf{a}=\left(a_{0}, \ldots, a_{m-1}\right)$ and $A(\mathbf{a})=\operatorname{circ}\left(a_{0}, \ldots, a_{m-1}\right)$. Then

$$
A(\mathbf{a})=F D(\mathbf{a}) F^{*},
$$

with

$$
D(\mathbf{a})=\operatorname{diag}\left(\lambda_{0}(\mathbf{a}), \lambda_{1}(\mathbf{a}), \ldots, \lambda_{m-1}(\mathbf{a})\right)
$$

and

$$
\lambda_{k}(\mathbf{a})=\sum_{\ell=0}^{m-1} a_{\ell} \omega^{k \ell}, \quad 0 \leq k \leq m-1 .
$$

Corollary 19. Let a defined as in Theorem 18 and consider

$$
\Lambda=\Lambda(\mathbf{a})=\left(\lambda_{0}(\mathbf{a}), \lambda_{1}(\mathbf{a}), \ldots, \lambda_{m-1}(\mathbf{a})\right) .
$$

Then,

$$
a_{k}=\frac{1}{m} \sum_{\ell=0}^{m-1} \lambda_{\ell} \omega^{-k \ell}, \quad 0 \leq k \leq m-1
$$


In [1], using the techniques in [15] for block matrices with circulant blocks, the following spectral result was proven.

Theorem 20. [1] Let $K$ be an algebraically closed field of characteristic 0 and suppose that $A=(A(i, j))$ is an mn-by-mn matrix partitioned into $n^{2}$ circulant blocks of $m$-by-m matrices, where for $1 \leq i, j \leq n$,

$$
A=(A(i, j)), A(i, j)=\operatorname{circ}(\mathbf{a}(i, j)),
$$

where

$$
\begin{array}{r}
\mathbf{a}(i, j)=\left(a_{0}(i, j), \ldots, a_{m-1}(i, j)\right), \\
a_{k}(i, j) \in K, \quad 1 \leq i, j \leq n, \quad 0 \leq k \leq m-1 .
\end{array}
$$

Then

$$
\sigma(A)=\bigcup_{k=0}^{m-1} \sigma\left(S_{k}\right)
$$

where

$$
S_{k}=\left(s_{k}(i, j)\right)_{1 \leq i, j \leq n}, s_{k}(i, j)=\sum_{\ell=0}^{m-1} a_{\ell}(i, j) \omega^{k \ell} .
$$

The next result is a direct consequence of Theorem 20 and of (12).

Corollary 21. [1] Consider $\mathbf{a}(u, v)=\left(a_{0}(u, v), \ldots, a_{m-1}(u, v)\right)$ and $A(u, v)=$ $\operatorname{circ}(\mathbf{a}(u, v))$. For $1 \leq \ell \leq m$, let matrix $S_{\ell}$, related to the circulant blocks $A(u, v)$ defined in (12). For $0 \leq k \leq m-1$, let

$$
\begin{aligned}
L_{k} & =\frac{1}{m} \sum_{\ell=0}^{m-1} S_{\ell} \omega^{-k \ell}, \\
L_{k} & =\left(\begin{array}{cccc}
a_{k}(1,1) & a_{k}(1,2) & \ldots & a_{k}(1, m) \\
\vdots & \vdots & \ddots & \vdots \\
a_{k}(m, 1) & a_{k}(m, 2) & \ldots & a_{k}(m, m)
\end{array}\right),
\end{aligned}
$$

In consequence, the matrix $A$ in (10) is nonnegative if and only if for all $0 \leq k \leq m-1$ the matrix $L_{k}$ is nonnegative and, in this case the matrix $S_{0}$ is nonnegative. 
From Theorem 20 the following inverse result was obtained.

Corollary 22. [1] Let $\left(S_{\ell}^{\prime}\right)_{\ell=0}^{m-1}$ be $m$ n-by-n complex matrices. Then there exists a matrix $A^{\prime}$ partitioned into blocks where each block is circulant and whose spectrum is given by

$$
\sigma\left(A^{\prime}\right)=\bigcup_{\ell=0}^{m-1} \sigma\left(S_{\ell}^{\prime}\right)
$$

The following notions will be used in the sequel.

Definition 23. [1] A matrix partitioned into blocks is called block permutative matrix when all its row blocks (up to the first one) are permutations of precisely its first row block.

The class of the block $X$-like permutative matrices is defined as follows.

Definition 24. Let $X=\left(x_{u v}\right)$ be the square matrix in (1). A block permutative matrix with circulant blocks $A=(\operatorname{circ}(\mathbf{a}(u . v))$ is a block X-like permutative matrix providing that, for all $1 \leq u, v \leq n$, the block circ $(\mathbf{a}(u, v))$ is the $(u, v)$-th block in $A$ if and only if $x_{u v}$ is the $(u, v)$-th entry in $X$.

Theorem 25. [1] Let $A$ be the matrix partitioned into blocks as defined in (10). For $0 \leq k \leq m-1$ let $S_{k}$ be the class of matrices related to $A$ defined in (12). The matrix $A$ is a block permutative matrix with circulant blocks if and only if the matrices $S_{k}$ are pairwise permutatively equivalent.

\section{An inverse problem related to block permutative matrices with circulant blocks}

In this section we study the Guo index to block permutative matrices with circulant blocks, which are a subclass of permutative matrices. Let $\mathbf{q}_{1}, \mathbf{q}_{2}, \ldots, \mathbf{q}_{m}$ denotes the canonical vectors

To our purpose we establish the following result.

Theorem 26. Let $E=\left(\varepsilon_{i j}\right)$ be an $n$-by-m matrix where the multiset $\{E\}$ formed by the entries of $E$ is closed under complex conjugation, the set of the entries in the first column of $E$ is a $X$-like permutative list and the sum of all row sums of $E$ is nonnegative. Suppose that $\varepsilon_{11}$ is positive and has the 
largest absolute value among the absolute values of entries of E. Moreover, for $1 \leq \ell \leq\left\lfloor\frac{m}{2}\right\rfloor$,

$$
E \mathbf{q}_{\ell+1}=\overline{E \mathbf{q}_{m-\ell+1}}
$$

that is, the entries of the $(m-\ell+1)$-th column of $E$ are the corresponding complex conjugate entries of the $(\ell+1)$-th column of $E$. Note that for $m=2 h$ the condition in (14) implies that the column $E q_{h+1}$ of $E$ has real entries. If

$$
\varepsilon_{11} \geq \Phi
$$

with

$$
\Phi=\max _{\substack{0 \leq k \leq m-1 \\ 0 \leq j \leq n-1}}-\left[\sum_{p=1}^{n-1} \varepsilon_{(p+1) 1}+\sum_{\ell=1}^{m-1} \sum_{p=0}^{n-1} \varepsilon_{(p+1)(\ell+1)} \omega^{-k \ell}-n \sum_{\ell=1}^{m-1} \varepsilon_{(p+1)(\ell+1)} \omega^{-k \ell}\right]
$$

Then $\{E\}$ is the spectrum of a nonnegative $X$-like block permutative matrix A whose blocks are circulant.

\section{Proof.}

By the conditions of the statement there exists a nonnegative permutative

$$
S_{0}:=\operatorname{per}_{X}\left(s_{00}, s_{10}, \ldots, s_{(n-1) 0}\right)
$$

whose spectrum is $\left\{E q_{1}\right\}$ (the set of the entries in $E q_{1}$ ). The condition in (14) implies that for $1 \leq \ell \leq\left\lfloor\frac{m}{2}\right\rfloor$ the $X$-like permutative matrices $S_{\ell}$ and $S_{m-\ell}$ whose spectrum are $\left\{E \mathbf{q}_{(\ell+1)}\right\}$ and $\left\{E \mathbf{q}_{(m-\ell+1)}\right\}$, respectively, are related by $\bar{S}_{\ell}=S_{m-\ell}$. For $1 \leq \ell \leq m-1$, suppose that

$$
S_{\ell}=\operatorname{per}_{X}(s(\ell)), \text { with } s(\ell)=\left(s_{0 \ell}, s_{1 \ell}, \ldots, s_{(n-1) \ell}\right),
$$

where

$$
s(\ell)^{T}=M^{-1} E \mathbf{q}_{\ell+1},
$$

then

$$
\begin{aligned}
& s_{0 \ell}=\frac{1}{n}\left[\sum_{p=1}^{n} \varepsilon_{p(\ell+1)}\right] \quad \text { and } \\
& s_{j \ell}=\frac{1}{n}\left[\sum_{p=1}^{n} \varepsilon_{p(\ell+1)}-n \varepsilon_{(j+1)(\ell+1)}\right], \quad 1 \leq j \leq n-1 .
\end{aligned}
$$


For $0 \leq k \leq m-1$, the entries of the $n$-by- $n X$-like permutative matrix can be obtained, using equation (9) and the entries of the sums

$$
L_{k}=\frac{1}{m} S_{0}+\frac{1}{m} \sum_{\ell=1}^{m-1} S_{\ell} \omega^{-k \ell},
$$

By Proposition 16 the linear combination of $X$-like permutative matrices are $X$-like permutative then matrices $L_{k}$ in (19) are $X$-like permutative. Suppose that

$$
L_{k}=\operatorname{per}_{X}\left(a_{0}(k), \ldots, a_{n-1}(k)\right) .
$$

From (19), for $0 \leq j \leq n-1$, the following holds

$$
a_{j}(k)=\frac{1}{m}\left(s_{j 0}+\sum_{\ell=1}^{m-1} \omega^{-k \ell} s_{j \ell}\right) .
$$

Using (17), for $1 \leq j \leq n-1$ we must have

$$
\begin{aligned}
0 \leq a_{j}(k) & =\frac{1}{m}\left(s_{j 0}+\sum_{\ell=1}^{m-1} \omega^{-k \ell} s_{j \ell}\right) \\
& =\frac{1}{m} \frac{1}{n}\left(\left[\varepsilon_{11}+\sum_{p=1}^{n-1} \varepsilon_{(p+1) 1}\right]+\sum_{\ell=1}^{m-1} \omega^{-k \ell}\left[\sum_{p=0}^{n-1} \varepsilon_{(p+1)(\ell+1)}-n \varepsilon_{(j+1)(\ell+1)}\right]\right) \\
& =\frac{1}{m n}\left(\varepsilon_{11}+\sum_{p=1}^{n-1} \varepsilon_{(p+1) 1}+\sum_{\ell=1}^{m-1} \sum_{p=0}^{n-1} \varepsilon_{(p+1)(\ell+1)} \omega^{-k \ell}-n \sum_{\ell=1}^{m-1} \omega^{-k \ell} \varepsilon_{(j+1)(\ell+1)}\right)
\end{aligned}
$$

for all $0 \leq k \leq m-1$. Therefore, the last condition implies the inequality in (15).

Remark 27. To computational aims it is worth note that the columns of the product

$$
L=\frac{1}{\sqrt{m}} M^{-1} E F^{*}
$$

are the first rows of the matrices $L_{0}, \ldots L_{m-1}$.

Now, one can formulate the following question. 
Problem 28. Which condition (or conditions) is (or are) necessary and sufficient for the existence of a nonnegative matrix, A X-like block permutative matrix with circulant blocks whose spectrum equals to $\{E\}$,? where $E=\left(\varepsilon_{i j}\right)$ is as in Theorem 20,

Let us consider the set

$$
\boldsymbol{P}=\{f:\{E\} \rightarrow\{E\}: f \text { is bijective }\}
$$

Definition 29. The function $f \in \boldsymbol{P}$ is said to be E-nonnegative spectrally stable (E-NNSS) if the matrix $E(f)=\left(f\left(\varepsilon_{i j}\right)\right)$ is such that $f\left(\varepsilon_{11}\right)$ has the maximum absolute value among $f\left(\varepsilon_{i j}\right)$, the first column of $E(f)$ is real and it is a X-like permutative list, the $(m-\ell+1)$-th column of $E(f)$ is the complex conjugate column of the $(\ell+1)$-th column of $E(f)$, and then

$$
E(f) \mathbf{q}_{(m-\ell+1)}=\overline{E(f)} \mathbf{q}_{(\ell+1)},
$$

for $1 \leq \ell \leq\left\lfloor\frac{m}{2}\right\rfloor$.

For instance:

1. The identity function of $E$ into $E$ is clearly $E$-NNSS.

2. $f:\{E\} \rightarrow\{E\}$ defined by

$$
f\left(\varepsilon_{i j}\right)=\left\{\begin{array}{ll}
\varepsilon_{i j} & j \neq 2 \\
\varepsilon_{i m} & j=2, \\
\varepsilon_{i 2} & j=m
\end{array} \text { and } j \neq m,\right.
$$

is $E$-NNSS.

3. $f:\{E\} \rightarrow\{E\}$ defined by

$$
f\left(\varepsilon_{i j}\right)=\bar{\varepsilon}_{i j} \text { for all } i, j
$$

is $E$-NNSS.

4. $f:\{E\} \rightarrow\{E\}$ defined by

$$
f\left(\varepsilon_{i j}\right)= \begin{cases}\varepsilon_{i j} & \text { if } j=1, \\ \bar{\varepsilon}_{i j} & \text { if } j \neq 1\end{cases}
$$

is E-NNSS. 
We denote by $\boldsymbol{P}^{*}$ the subset of $\boldsymbol{P}$ formed by all $E$-NNSS bijections of $E$. Note that if $f \in P^{*}$ then $f\left(\varepsilon_{11}\right)=\varepsilon_{11}$.

Theorem 30. Let $E=\left(\varepsilon_{i j}\right)$ be an $n$-by-m matrix such that its first row is a $X$-like permutative spectrum. Moreover, the multiset $\{E\}$ formed by the entries of E satisfies the conditions in Theorem [26. The multiset $\{E\}$ is the spectrum of a block $X$-like permutative matrix $A$ whose blocks are circulant if and only if

$$
\varepsilon_{11} \geq \min _{f \in \boldsymbol{P}^{*}} \max \{\Theta: 0 \leq k \leq m-1,0 \leq j \leq n-1\}
$$

where,

$\Theta=-\left[\sum_{p=1}^{n-1} f\left(\varepsilon_{(p+1) 1}\right)+\sum_{\ell=1}^{m-1} \sum_{p=0}^{n-1} f\left(\varepsilon_{(p+1)(\ell+1)}\right) \omega^{-k \ell}-n \sum_{\ell=1}^{m-1} \omega^{-k \ell} f\left(\varepsilon_{(j+1)(\ell+1)}\right)\right]$.

Proof. Following the same steps of the above proof this time replacing $\varepsilon_{i j}$ by $f\left(\varepsilon_{i j}\right)$ we arrive at the inequality in (15). After taking the minimum when the function $f$ vary into $\boldsymbol{P}^{*}$, the inequality (20) is obtained.

Example 31. Let consider

$$
E=\left(\begin{array}{ccc}
23.9 & i & -i \\
-3 & 1-7 i & 1+7 i \\
0 & -3+i & -3-i
\end{array}\right)
$$

The nonnegative $X$-like permutative matrix per $_{X}(6.9667,9.9667,6.9667)$ has the set formed by the components of $E \mathbf{q}_{1}$ as spectrum. In addition we have

$$
\begin{aligned}
& L_{0}=\operatorname{per}_{X}(1.8778,2.2111,3.8778) \\
& L_{1}=\operatorname{per}_{X}(1.5822,6.9570,0,0048) \\
& L_{2}=\operatorname{per}_{X}(3.5067,0.7986,3.0840)
\end{aligned}
$$

In consequence, the circulant blocks are 


$$
\begin{aligned}
& A_{0}=\operatorname{circ}(1.8778,1.5822,3.5067) \\
& A_{1}=\operatorname{circ}(2.2111,6.9570,0.7986) \\
& A_{2}=\operatorname{circ}(3.8778,0,0048,3.0840)
\end{aligned}
$$

Therefore, the $X$-like block permutative matrix

$$
\left(\begin{array}{ccc}
A_{0} & A_{1} & A_{2} \\
A_{1} & A_{0} & A_{2} \\
A_{2} & A_{1} & A_{0}
\end{array}\right)
$$

has spectrum the multiset $\{E\}$.

Example 32. Let consider

$$
E_{1}=\left(\begin{array}{cccc}
5 & -3 & -2 & -3 \\
2 & 1 & 2 & 1
\end{array}\right)
$$

The nonnegative $X$-like permutative matrix per $_{X}(3.5,1.5)$ has the set formed by the components of $E \mathbf{q}_{1}$ as spectrum. In addition we have the matrices

$$
L_{0}=\operatorname{per}_{X}(1.5,0), L_{1}=\operatorname{per}_{X}(2,2), L_{2}=\operatorname{per}_{X}(2.5,2), L_{3}=\operatorname{per}_{X}(2,2) \text {. }
$$

In consequence, we obtain

$$
A_{0}=\operatorname{circ}(1.5,2,2.5,2), A_{1}=\operatorname{circ}(0,2,2,2) .
$$

Therefore, the $X$-like block permutative matrix

$$
\left(\begin{array}{ll}
A_{0} & A_{1} \\
A_{1} & A_{0}
\end{array}\right)
$$

has spectrum the multiset $\left\{E_{1}\right\}$.

Example 33. Let consider

$$
E_{2}=\left(\begin{array}{cccc}
2.5 & 0.25 i & 0 & -0.25 i \\
-1 & 0.5-i & 0 & 0.5+i
\end{array}\right)
$$


thus, it is obtained

$$
\begin{gathered}
L_{0}=\operatorname{per}_{X}(0.25,0.375), L_{1}=\operatorname{per}_{X}(0,0.75), \\
L_{2}=\operatorname{per}_{X}(0.125,0.5) L_{3}=\operatorname{per}_{X}(0.375,0.125) .
\end{gathered}
$$

In consequence,

$$
A_{0}=\operatorname{circ}(0.25,0.0,0.125,0.375), A_{1}=\operatorname{circ}(0.375,0.75,0.5,0.125) .
$$

Therefore, the $X$-like block permutative matrix

$$
\left(\begin{array}{cc}
A_{0} & A_{1} \\
A_{1} & A_{0}
\end{array}\right)
$$

has spectrum the multiset $\left\{E_{2}\right\}$.

$B y$ consider in $E_{2}(1,1)=2.49$ in a place of $E_{2}(1,1)=2.5$ it is obtained

$$
\begin{aligned}
& L_{0}=\operatorname{per}_{X}(0.2488,0.3738) \\
& L_{1}=\operatorname{per}_{X}(-0.0012,0.7488) \\
& L_{2}=\operatorname{per}_{X}(0.1238,0.4987) \\
& L_{3}=\operatorname{per}_{X}(0.3738,0.1238) .
\end{aligned}
$$

Therefore, the $X$-like block permutative matrix that we can construct is no nonnegative.

Example 34. Let consider

$$
E_{3}=\left(\begin{array}{ccc}
4 & 1 & 1 \\
-1 & -2.5 & -2.5
\end{array}\right)
$$

Thus are obtained the matrices

$$
L_{0}=\operatorname{per}_{X}(0,2), L_{1}=\operatorname{per}_{X}(0.75,0.25), L_{2}=\operatorname{per}_{X}(0.75,0.25) .
$$

In consequence,

$$
A_{0}=\operatorname{circ}(0,0.75,0.75), A_{1}=\operatorname{circ}(2,0.25,0.25) .
$$

Therefore, the $X$-like block permutative matrix

$$
\left(\begin{array}{ll}
A_{0} & A_{1} \\
A_{1} & A_{0}
\end{array}\right)
$$

has spectrum the multiset $\left\{E_{3}\right\}$. Considering the multiset formed by the entries of $E_{1}, 4$ is the least Perron root that can be considered because the trace becomes negative if the spectral radius is diminished.

\section{References.}




\section{References}

[1] E. Andrade, C. Manzaneda, H. Nina, M. Robbiano. Block matrices and Guo's Index for block circulant matrices with circulant blocks. Paper Accepted.

[2] A. Berman, R. J. Plemmons, Nonnegative matrices in the Mathematical Sciences, SIAM Publications, Philadelphia, 1994.

[3] A. Borobia. On nonnegative eigenvalue problem, Lin. Algebra Appl. 223/224 (1995): 131-140, Special Issue honoring Miroslav Fiedler and Vlastimil Pták.

[4] S. Friedland, On an inverse problem for nonnegative and eventually nonnegative matrices, Israel T. Math. 1, 29 (1978): 43-60.

[5] M. Fiedler, Eigenvalues of nonnegative symmetric matrices, Lin. Algebra Appl. 9 (1974): 119-142.

[6] W. Guo, Eigenvalues of nonnegative matrix, Linear Algebra and its Applications 266 (1997): 261-270.

[7] C. R. Johnson, Row stochastic matrices similar to doubly stochastic matrices, Lin. and Multilin. Algebra 2 (1981): 113-130.

[8] C. Johnson, T. Laffey, R. Loewy, The real and symmetric nonnegative inverse eigenvalue problems are different, Proc. Amer. Math Soc.,12, 124 (1996): 3647-3651.

[9] H. Karner, J. Schneid, C. W. Ueberhuber, Spectral decomposition of real circulant matrices. Lin. Algebra Appl. 367 (2003): 301-311.

[10] T. Laffey, Extreme nonnegative matrices, Lin. Algebra Appl. 275/276 (1998): 349-357. Proceedings of the sixth conference of the international Linear Algebra Society (Chemnitz, 1996).

[11] T. Laffey, Realizing matrices in the nonnegative inverse eigenvalue problem, Matrices and group representations (Coimbra, 1998), Textos Mat. Sér. B, 19, Univ. Coimbra, Coimbra, (1999): 21-31.

[12] T. Laffey, H. Šmigoc, Nonnegative realization of spectra having negative real parts, Linear Algebra Appl., 384 (2004): 199-206. 
[13] R. Loewy, J. J. Mc Donald, The symmetric nonnegative inverse eigenvalue problem for $5 \times 5$ matrices, Linear Algebra Appl. 393 (2004): 275-298.

[14] R. Loewy, D. London, A note on an inverse problem for nonnegative matrices, Lin. and Multilin. Algebra 6, 1 (1978/79): 83-90.

[15] C. Manzaneda, E. Andrade, M. Robbiano, Realizable lists via the spectra of structured matrices, Lin. Algebra Appl. 534 (2017): 51-72.

[16] P. Paparella, Realizing Suleı̆manova-type spectra via permutative matrices, Electron. J. Linear Algebra, 31 (2016): 306-312.

[17] H. Šmigoc, The inverse eigenvalue problem for nonnegative matrices, Linear Algebra Appl. 393 (2004): 365-374.

[18] H. Šmigoc, Construction of nonnegative matrices and the inverse eigenvalue problem, Lin. and Multilin. Algebra 53, 2 (2005): 85-96. 